\title{
Preparation and Research of Hydrophilic Polyurethane Modified by Folate-Lactose
}

\author{
Liudmyla Kulyk, Iryna Gladyr, Rita Rozhnova*, Dmytro Kuliesh, Nataliia Galatenko, \\ Larisa Narazhaiko
}

Department of Polymers of Medical Appointment, Institute of Macromolecular Chemistry of National Academy of Science of Ukraine, Kyiv, Ukraine

\author{
Email address: \\ lyudmila.makeeva@gmail.com (L. Kulyk),rozhnovarita@gmail.com (R. Rozhnova),d_kulesh@ukr.net(D. Kuliesh), \\ politoks@merlin.net.ua(N. Galatenko) \\ ${ }^{*}$ Corresponding author
}

\section{To cite this article:}

Liudmyla Kulyk, Iryna Gladyr, Rita Rozhnova, Dmytro Kuliesh, Nataliia Galatenko, Larisa Narazhaiko. Preparation and Research of Hydrophilic Polyurethane Modified by Folate-Lactose. American Journal of Polymer Science and Technology.

Vol. 5, No. 2, 2019, pp. 63-72. doi: 10.11648/j.ajpst.20190502.14

Received: May 21, 2019; Accepted: June 26, 2019; Published: July 9, 2019

\begin{abstract}
The biological active polyurethane (PU) based on diisocyanate prepolymer and folate-lactose (FL) was synthesized. Physical, mechanical and thermophysical properties of polymeric films were investigated. It was found that the value of the strength at break and elongation of PU with FL are higher by $83 \%$ and $22 \%$ than the corresponding physical and mechanical properties of the PU with D-lactose. According to the obtained results, the characteristic viscosity and water absorption of PU with FL are higher by $44 \%$ and $34 \%$ for the parameters of PU containing D-lactose. It has been established that prepeared PU are single-phase. The glass transition temperature is $247 \mathrm{~K}$ for PU with FL and $245 \mathrm{~K}$ for PU with D-lactose. At the same time, FL introduction into the polymer matrix causes decreasion of the heat jump value $(\Delta \mathrm{Cp})$ to $0,1551 \mathrm{~J} / \mathrm{g}\left({ }^{\circ} \mathrm{C}\right)$ for PU with FL sample and $0,2911 \mathrm{~J} / \mathrm{g}\left({ }^{\circ} \mathrm{C}\right)$ for PU with D-lactose sample. Biodegradation of synthesized materials was investigated after incubation in physiological solution $(0.9 \% \mathrm{NaCl})$ and biological medium 199 for 180 days. According to research results, polyurethane, modified by folate-lactose, retains sufficient performance for use within 6 months in conditions close to the human body. Biological activity and biocompatibility of synthesized polyurethane modified with FL were confirmed by tissue culture and histological methods.
\end{abstract}

Keywords: Folic Acid, Polyurethane, Lactose, Folate-Lactose, Biodegradation, Biocompatibility

\section{Introduction}

In medical practice, biologically active polymer-based materials based on polyurethanes are widely used due to their performance characteristics, biocompatibility and the ability to vary widely in properties depending on the composition, conditions of synthesis and modification [1-3].

The main approaches used to create biologically active polymeric materials are to provide biological activity by introducing a drug as a filler, by binding the drug to a polymeric matrix by covalent bonding or immobilizing the dosage form via a biodegradable spacer.

To provide biological activity to such materials, immobilization of medicinal substances, vitamins, organometallic compounds, and other is carried out on the selected polymer carrier. As a result, obtained materials have the ability to prolong the release of the biologically active substance directly to the place of use, thereby displaying its therapeutic effect. Biologically active polymeric materials of this type of medical purpose can be considered as systems with addressable therapeutic influence (drug delivery systems).

From the point of view of the biologically active compound, the prolonged form of which in the polymer carrier is capable of providing stimulation of the regenerative process, deserves attention folic acid - N-pteroyl-Lglutamic acid (FA). Since FA in the body manifests its biological activity through its conversion into tetrahydrofolic acid (by addition of hydrogen atoms to carbon atoms and 
nitropteroyl residue) [4], the introduction of FA into a polymer matrix by conjugation through a functional group, that does not belong to pteroyl cycle, allows you to obtain a material with its own biological activity, which can stimulate tissue regeneration.

Nowadays, there are works on the creation of drug delivery systems with folic acid [5-10]. As example, the folate-conjugated bioglass was synthesized by the authors [7], which is a bioactive, biodegradable and hydrophilic composition based on ferric oxide (III), sodium, calcium, silicon (IV) and phosphorus (V). Folic acid conjugation was carried out through the carboxylic group of folic acid by forming a polyethylene-bis-amine-folic acid complex in the presence of N,N'-dicyclohexylcarbodiimide and pyridine, as a reaction catalyst, followed by its immobilization on the bioglass surface using aminosilan as a coupling agent. The medical purpose of bioglass is the carrier of drugs and genes for use in magnetic induced hyperthermia of tumor processes.

Folate conjugated magnetite [8] is known as a system for addressing the delivery of therapeutic drugs with controlled release of medicinal substance at the place of pathology. Synthesis was accomplished by the functionalization of magnetite with polyethylene glycol, followed by conjugation with folic acid through its carboxyl group. But the above listed biologically active materials are intended for use in the treatment of pathological processes.

Folate-modified spermine- and acetal-modified dextran particles as a promising drug delivery system for a tumor cell targeted photodynamic therapy were obtained in [9]. In [10], perspective drug carrier, based on the formation of carbodiimide compounds between carboxyl and primary amines, hollow microspheres arising from the folic acid grafted onto the surface of the modified hydroxyapatite was described.

Biologically active folate-based polyurethane ureas (PUU), proposed for use as film materials for the treatment of wounds and burns, are known [11], in which folic acid is introduced into the structure of the polymer material through an amide bond due to the interaction of the amino group of folic acid with the NCO groups of the polymer matrix. As part of the PUU, there is also a part of the FA, that is immobilized physically and provides the biological activity of the developed material.

There are PUU, which contain in their structure folateferrocene conjugate and exhibit biological activity in the conditions of tissue culture and during implantation into experimental animals [12]. The above listed biologically active polymeric materials are biocompatible, stable to biodegradation, capable of withstanding sterilization without altering properties and stimulate regenerative processes.

Development of new ways of immobilization of folic acid on a polyurethane carrier is relevant, one of which is modification of folic acid with disaccharide through biodegradable azomethine bond. Such an approach will increase the hydrophilicity of the polymer matrix by introducing into the polymer carrier a hydrophilic component of natural origin and will enable the prolonged release of the drug to the place of the tissue damage and maintain its necessary concentration due to the hydrolysis of the azomethine bond.

Thus, the purpose of the work is to synthesis of hydrophilic polyurethane modified by folate-lactose and the study of physical, mechanical and thermophysical properties, biological activity and biocompatibility of the obtained polyurethane.

\section{Experimental}

\subsection{Materials}

Folic acid (FA) (Acros, 96,6\%) and D-lactose $(\geq 99 \%$, Merck) were dried before use at the temperature $398 \mathrm{~K}$ during 3 hours. Triethylamine (Merck, 99,9\%) was used to block acid groups of FA.

Polyoxypropylene glycol (POPG) (Rokopol, M 1000) for polyurethane preparation was obtained from PPC Rokita SA (Poland).

Toluylendiisocyanate (TDI, 86,7\%) was acquired for polyurethane preparation from Merck. It contained a mixture of 2,4-, 2,6-isomers per ratio $80 / 20 \%$ w.).

N,N'- Dimethylacetamide (DMAA, 99,8\%) was obtained from Merck.

Dimethylsulfoxide (DMSO, 99,8\%), diethylether (Ph Eur), chloroform (Ph Eur), acetone ( $\geq 99 \%)$ were purchased from Chimlaborreactive Ltd. (Kyiv, Ukraine).

All other chemical reagents and solvents that were used in the work were analytically pure.

To study the biodegradation of the obtained polyurethanes, a physiological solution $(0,9 \% \mathrm{NaCl}, \mathrm{Ph}$ Eur) was used, which was purchased from Pharmacia Ltd (Kyiv, Ukraine) and biological medium 199 (Pharmacia Ltd, Kyiv, Ukraine).

\subsection{Study Methods}

\subsubsection{The Method of Incubation in Biological Medium 199}

Samples of PU in the form of strips in the size of $0.5 \times 5.0$ $\mathrm{cm}$ were placed in sterile tubes, poured with $25 \mathrm{ml}$ of the model biological medium and kept in a thermostat at a temperature of $310 \mathrm{~K}$ for 1,3 and 6 months. Solutions of the model environment changed daily. The biological medium 199 (BM 199), which is a complex mixture of proteins, amino acids, carbohydrates, fats, salts, hormones, enzymes and soluble gases $(\mathrm{pH}=7,4)$, and physiological solution $(0,9 \% \mathrm{NaCl})$ were used as model medium. After a certain period of residence in the model medium, the samples were removed, washed with distilled water and dried to constant mass at the room temperature.

\subsubsection{Fourier Transforms Infrared (ATR FT-IR)}

Infrared absorption spectra were taken on Bruker infrared spectrometer with Tensor-37 Fourier transform by the method of disturbed full internal reflection in the region of $600-4000 \mathrm{~cm}^{-1}$, using the prism-trapezoid of the diamond crystal (the number of reflections $N=1$, the angle of 
incidence $\varphi=39^{\circ}$ ).

\subsubsection{Differential Scanning Calorimetry (DSC)}

The thermophysical properties of synthesized PU with folate-lactose and lactose were studied by DSC using the instrument Q2000 (TA Instruments, USA) in air atmosphere in temperature range from 183 to $473 \mathrm{~K}$, heating rate 20 $\mathrm{deg} / \mathrm{min}$. In order to level the influence of the conditions of synthesis of samples of warming carried out 2 times. The thermograms of the samples under study are shown in Figure 5.

\subsubsection{Mechanical Tests}

The tensile strength $(\sigma, \mathrm{MPa})$ and relative elongation $(\varepsilon, \%)$ of the synthesized polymer films were determined using 2166 P-5 tension testing machine at a clamping speed of (50 \pm 5$)$ $\mathrm{mm} / \mathrm{min}$ the rate of fixation of results is $0.01 \mathrm{sec}$ in accordance with GOST 25.601.

\subsubsection{Absorption Test}

Water absorption $(W, \%)$ of samples was determined in the distilled water at $T(296 \pm 2) \mathrm{K}$ for 24 hours under the GOST 2650 .

Water absorption was calculated according to the formula:

$$
W=\frac{m_{2}-m_{1}}{m_{2}} \times 100
$$

where $m_{l}$ - sample mass before immersion in water, $\mathrm{g}$.

$m_{2}$ - sample mass after keeping in water for 24 hours, $\mathrm{g}$.

The results of the research are presented in the Table 1 .

\subsubsection{Viscometry}

The characteristic viscosity $(\eta, \partial \mathrm{l} / \mathrm{g})$ was determined by the viscometric method using the Ostwald viscometer with a capillary diameter of $0,62 \mathrm{~mm}$. The characteristic viscosity of polymers was determined by graphic extrapolation of the specific viscosity of polymer solutions in DMAA at a temperature of $(296 \pm 2) \mathrm{K}$ in the range of $0.5-1.0 \%$ to zero concentration. The specific viscosity of $1 \%$ polymer solutions was determined in N,N'-dimethylacetamide at (296 $\pm 2) \mathrm{K}$.

\subsubsection{Tissue Culture Methods}

The method of tissue culture is a model test system in a toxicological experiment. The subcutaneous tissue of white laboratory rats was used as source of cells for research, due to the fact, that, under cultivation conditions, it causes the growth of fibroblast and fibroblast-like elements. BM 199 was used as a model medium for tissue cultures. Biological activity of PU was studied in cultures by explantation in plasma clot (solid phase) in Carrel vials with the addition of a nutrient mixture (liquid phase). The subcutaneous fat of white male rats 3 months old was used as the implantation material. Samples of investigated polymeric materials in the size of $0.5 \times 0.5 \mathrm{~cm}$ were placed in vials, then an embryonic extract was added and a plasma clot (solid phase) was obtained. After solid phase formation (10-15 min.), a nutrient biological medium 199 and a serum of cattle (liquid phase) were added. The cultivation was carried out at $310 \mathrm{~K}$, the liquid phase was changed every three days. Vials with subcutaneous-fatty tissue explants without the addition of polymer specimens were used as control samples. The cultures were incubated at $310 \mathrm{~K}$.

In order to standardize the nature of the growth of cultures, their zones were classified as compact, mesh and zone of migratory cells, the criterion for distinguishing them was the nature of the location of growing fibroblast cells. To determine the effect of the samples under study on the growth of tissue culture, the samples were taken at the $3^{\mathrm{d}}$ day of cultivation, when the migration of fibroblast elements in the vials began. Studies of growth and development of cellular elements of subcutaneous fat of white rats were performed at 3, 7, 10, 14 and 21 days of cultivation. The cultures were examined under a microscope in the native state and when stained with hematoxylin-eosin and picro fuchsinVan Giezon.

\subsubsection{Histological Method}

Histological studies were conducted to study the reaction of the surrounding tissues of the body when implantation of samples of polymeric materials.

Subcutaneous implantation of samples of PU (size $10 \times 10$ $\times 2 \mathrm{~mm}$ ) in the region of the back of white male rats was carried out under anesthesia, in conditions of asepsis. Animals were withdrawn from the experiment by overdose of ether at 7, 14, 30 and 90 days after the operation. Implants were removed along with the surrounding tissues. For morphological analysis after standard histological treatment (fixation in 10\% formalin solution, dehydration in increasing concentrations of ethanol, pouring into paraffin) of the connective tissue capsule formed around the implanted material, cut-offs of 10-15 $\mu \mathrm{m}$ thickness were made that were stained with hematoxylin and eosin, and microscopic analysis was performed. The evaluation of biocompatibility of polymeric materials was carried out by histological analysis using microscopy (Micmed-2 microscope), microphotography was performed using a video glass ScienceLab 3.0.

\section{Results and Discussion}

\subsection{Preparation of Polyurethanes Are Modified by Folate-Lactose}

Synthesis of folate-lactose (FL) was performed per molar ratio of FA: Lactose $=1: 1$ in the medium of DMSO at $(353 \pm$ 5) $\mathrm{K}$ during 4 hours with stirring by the technique as described in [13].

Synthesis of the film materials, based on diisocyanate prepolymer (DPP) and folate-lactose was performed in 2 stages (Figure 2). At the first stage, the synthesis of DPP was performed on the basis of POPG and TDI for the molar ratio of POPG:TDI $=1: 2\left(\mathrm{NCO}_{\text {free }}-6.23 \%\right.$, determined by the titrimetric method [14]). At stage 2 polyaddition reaction between DPP and FL at molar ratio 1:1 was performed. The reaction was monitored by IR spectroscopy by determining 
the content of free NCO groups - until complete conversion ( $\sim 60 \mathrm{~min})$. The reaction mixture was poured onto teflon pads and dried in a drying cabinet at a temperature of $(343 \pm 5) \mathrm{K}$ for 72 hours to constant weight. Polyurethane was obtained in the form of transparent brown films, soluble in DMAA and DMFA.
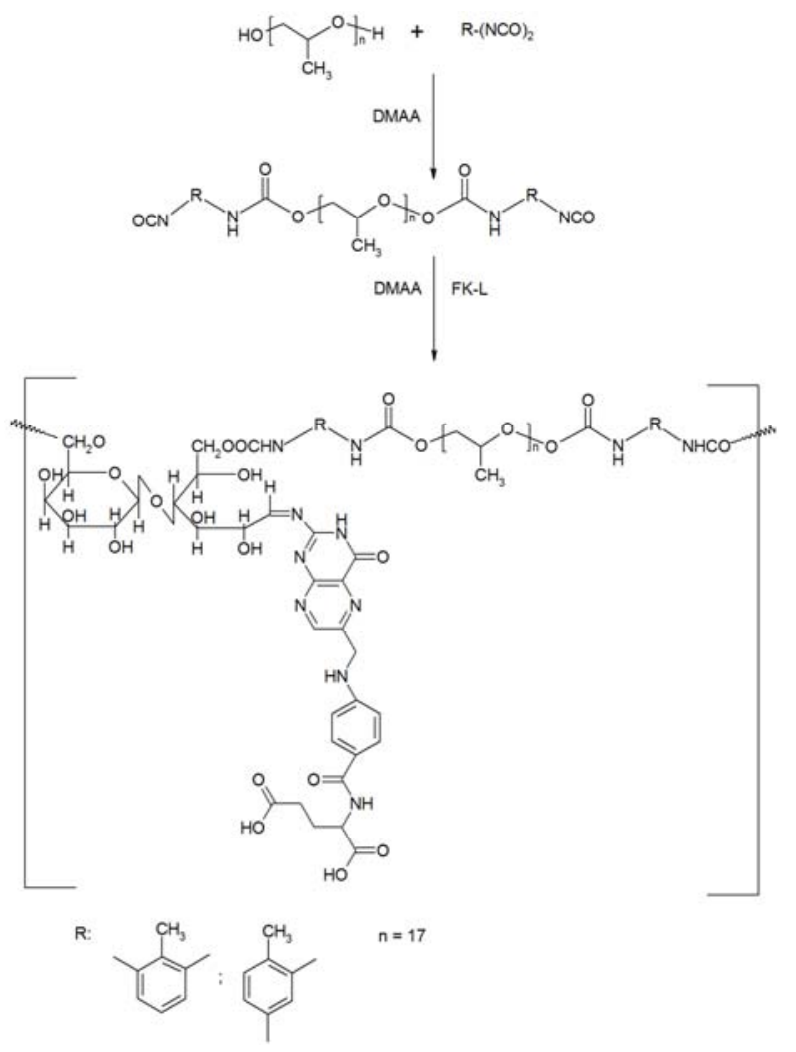

Figure 1. Scheme of polyurethane containing folate-lactose synthesis.

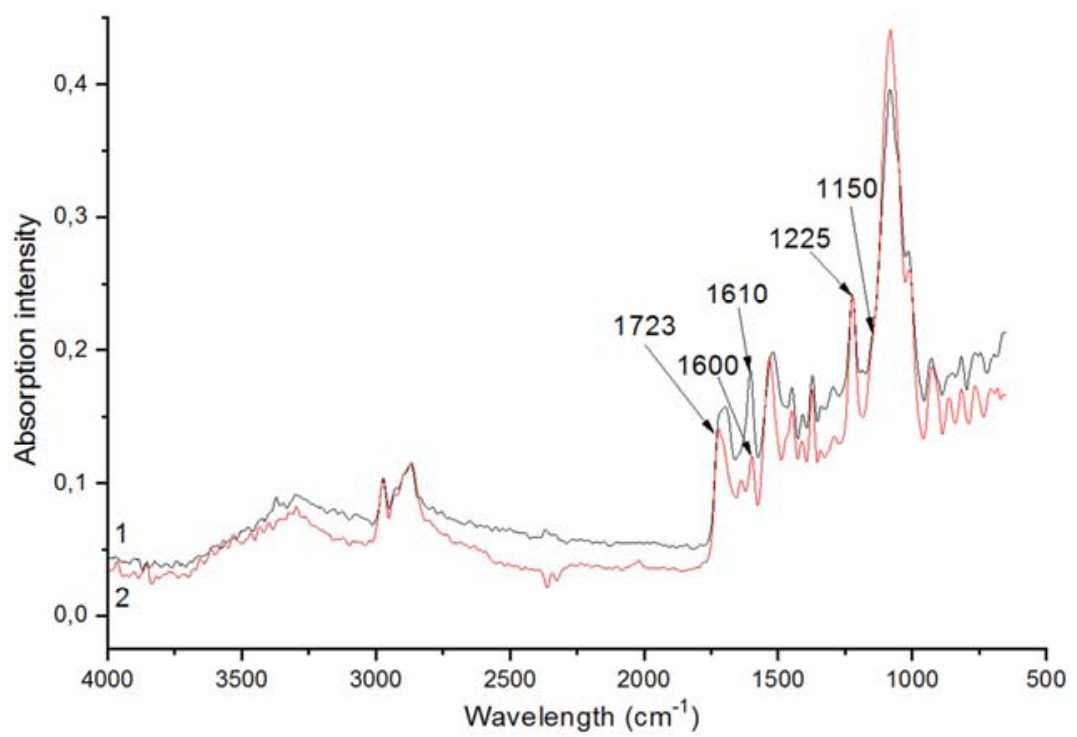

Figure 3. IR-spectra: 1 - polyurethane containing folate-lactose; 2 - polyurethane containing D-lactose.

IR-spectrum polyurethane containing folate-lactose (Figure, curve 1) is characterized by the presence of stripes $v_{\mathrm{C}=\mathrm{O}}$ at $1723 \mathrm{~cm}^{-1}, \delta_{\mathrm{C}-\mathrm{N}}$ at $1610 \mathrm{~cm}^{-1}, \delta_{\mathrm{C}-\mathrm{O}-\mathrm{C}}$ at 1225 and 1150
Polyurethane based on diisocyanate prepolymer and Dlactose was obtained in 2 similar stages as it was described for polyurethane containing folate-lactose in order to be used as a sample of comparison. The polymeric material was obtained in the form of transparent yellow films, soluble in amide solvents with general formula shown on Figure 2.

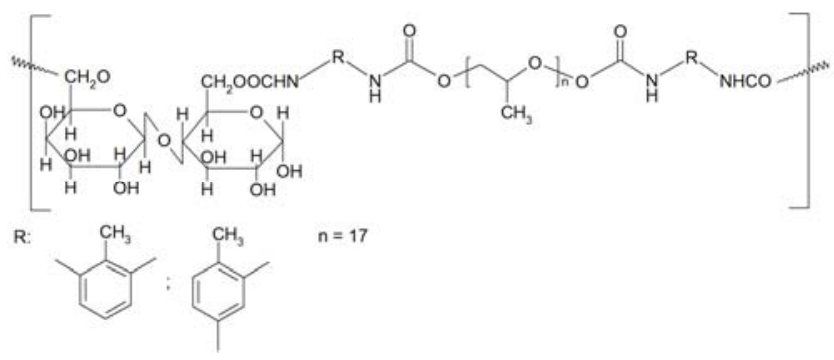

Figure 2. A fragment of the formula of polyurethane, containing D-lactose.

It was found, that polymeric material with folate-lactose has such characteristics: tensile strength $-10 \mathrm{MPa}$, relative elongation $-64 \%$, intrinsic viscosity - 0,18 dl/g, moisture absorption $-13 \%$ and they are much higher than appropriate characteristics of polyurethane, modified by D-lactose.

\subsection{FTIR IR-Spectroscopy}

Obtained polymeric materials were investigated by the method of IR-spectroscopy (Figure 3). Infrared spectra of polymer films were recorded on infrared spectrometer "Bruker" with Fourier transformation "Tensor-37" for reflection in the region of $600-4000 \mathrm{~cm}^{-1}$. The assignment of the absorption bands was carried out according to the data of works $[15,16]$. $\mathrm{cm}^{-1}$ of urethane fragment. At IR-spectrum of polyurethane containing D-lactose (Figure 3, curve 2) band of deformation vibrations $\delta_{\mathrm{C}-\mathrm{N}}$ shifted to higher frequencies $\left(1600 \mathrm{~cm}^{-1}\right)$. 
Such a profile of the streams of synthesized materials confirms the structure of polymers and the nature of the reaction proceeding described above.

\subsection{Mechanical and Absorption Test}

In order to determine the influence of modification of Dlactose with folic acid on the performance characteristics of polymeric materials, water absorption, viscosimetric, physical and mechanical properties of synthesized polyurethanes were studied (Table 1). The value of the strength at break and elongation of polymer material samples with folate-lactose are higher by $83 \%$ and $22 \%$ than the corresponding physical and mechanical properties of the polymer material with D-lactose. The data of viscosimetry and the study of hydrophilicity - the indexes of the characteristic viscosity and water absorption of polyurethane containing folate-lactose are higher by $44 \%$ and $34 \%$ for the parameters of polyurethane containing D-lactose.

Table 1. Parameters of tensile strength, relative elongation, characteristic viscosity and water absorption of synthesized PU.

\begin{tabular}{lllll}
\hline Samples of PU & $\boldsymbol{\eta}_{\text {character }}, \partial \mathbf{d} / \mathbf{g}$ & $\boldsymbol{\sigma}, \mathbf{M P a}$ & $\mathbf{\varepsilon , \%}$ & $\mathbf{W}, \mathbf{\%}$ \\
\hline PU with D-lactose & 0,10 & $1,65 \pm 0,07$ & $49,56 \pm 5,58$ \\
PU with FL & 0,18 & $10,24 \pm 0,24$ & $63,69 \pm 0,61$ \\
\end{tabular}

\subsection{Termophysical Test}

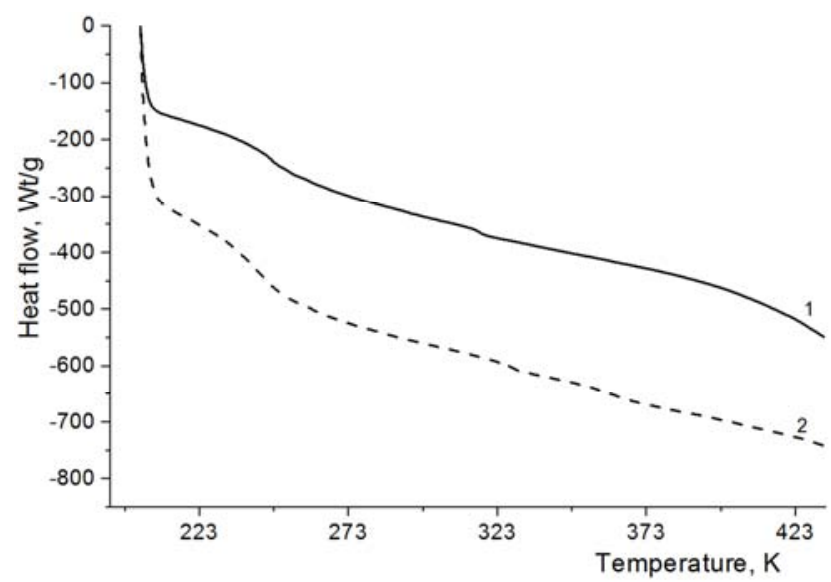

On the thermograms of the studied PU, one temperature transition and, respectively, one glass transition temperature were observed $\left(\mathrm{T}_{\mathrm{g}}\right)$ (Figure 4$)$. Thus, the studied systems are single-phase. The glass transition temperature is $247 \mathrm{~K}$ for PU with FL and $245 \mathrm{~K}$ for PU with D-lactose. At the same time, folate-lactose introduction into the polymer matrix causes decreasion of the heat jump value $\left(\Delta \mathrm{C}_{\mathrm{p}}\right)$ to 0,1551 $\mathrm{J} / \mathrm{g}\left({ }^{\circ} \mathrm{C}\right)$ for PU with FL sample and $0,2911 \mathrm{~J} / \mathrm{g}\left({ }^{\circ} \mathrm{C}\right)$ for PU with D-lactose sample.

\subsection{Biodegradation Study in vitro}

Studies of PU biodegradation with FL were performed after incubation in physiological solution $(0.9 \% \mathrm{NaCl})$ and biological medium 199 for 180 days. The results of the research are presented in the Table 2.

Figure 4. Thermograms of PU samples:PU with FL (1); PU with D-lactose (2).

Table 2. Properties of PU after incubation in a physiological solution and BM 199.

\begin{tabular}{|c|c|c|c|c|c|c|c|c|}
\hline \multirow{2}{*}{ Incubation term } & \multicolumn{4}{|c|}{ DPP-lactose } & \multicolumn{4}{|c|}{ DPP-FL } \\
\hline & $\eta, \partial \mathrm{l} / \mathrm{g}$ & $\sigma, \mathbf{M P a}$ & $\varepsilon, \%$ & Weight loss, $\%$ & $\eta, \partial \mathrm{l} / \mathrm{g}$ & $\sigma, \mathrm{MPa}$ & $\varepsilon, \%$ & Weight loss, $\%$ \\
\hline before & 0,15 & 1,7 & 50 & - & 0,21 & 10,2 & 64 & - \\
\hline \multicolumn{9}{|c|}{ Physiological solution } \\
\hline 14 days & 0,17 & 1,2 & 103 & 17,7 & 0,16 & 10,3 & 53 & 9,5 \\
\hline 1 month & 0,17 & 1,3 & 123 & 18,5 & 0,11 & 10,6 & 57 & 12,0 \\
\hline 3 month & 0,17 & 3,0 & 186 & 21,7 & 0,33 & 12,1 & 60 & 13,0 \\
\hline 6 month & 0,15 & 2,4 & 116 & 23,6 & 0,26 & 14,2 & 37 & 13,5 \\
\hline 14 days & 0,24 & 1,4 & 106 & 17,7 & 0,14 & 9,4 & 36 & 18,8 \\
\hline 1 month & 0,29 & 1,5 & 163 & 18,0 & 0,17 & 9,2 & 40 & 16,8 \\
\hline 3 month & 0,14 & 2,6 & 115 & 22,1 & 0,18 & 7,2 & 51 & 25,6 \\
\hline 6 month & 0,15 & 2,7 & 123 & 21,2 & 0,23 & 9,6 & 41 & 24,9 \\
\hline
\end{tabular}

As a result of the research, it was found that the polymeric materials obtained on the basis of DPP and lactose lose up to $23.6 \%$ of the weight from the initial after incubation in BM 199 and physiological solution. At the same time, there is a gradual increase in the tensile strength of samples. Specific viscosity and relative elongation do not undergo significant changes compared to rates before incubation. The nature of the change in the properties of lactose-containing polymeric samples practically does not depend on the medium of incubation.
Polyurethanes obtained on the basis of DPP and folatelactose inherent in the different nature of the course of biodegradation depending on the environment of incubation. For DPP-FL samples after 6 months of incubation in physiological solution, a $13.5 \%$ reduction in weight compared to the initial one was observed, while samples sustained in the BM 199 lost $25.6 \%$ by weight. The gradual slight increase in the strength of polyurethane samples in vitro is probably due to the redistribution of hydrogen intermolecular and intra-molecular bonds under the influence 
of model environments, along with the biodegradation of the polymer matrix. Thus, according to the obtained results, the synthesized polymeric materials maintain sufficient physical and mechanical characteristics in the conditions close to the human body to 6 months.

\subsection{Tissue Culture Studies}

Biological activity and biocompatibility of new polyurethane were investigated by tissue culture method.

The study was conducted in tissue culture of white rats subcutaneous fat. The effect of the PU samples on the growth and development of fibroblastic elements that are key cellular

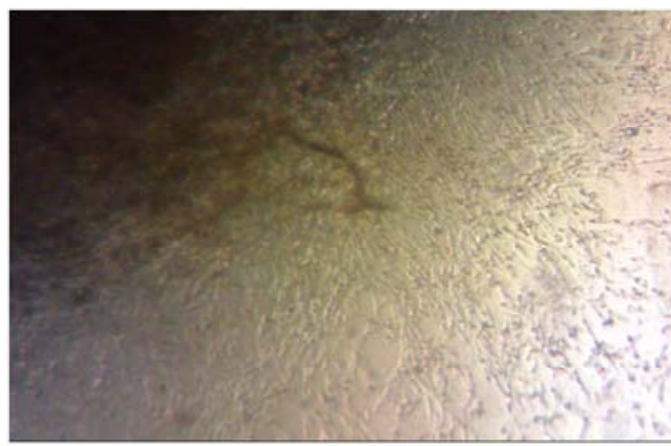

elements participating in the process of healing tissue injuries was investigated.

Research of the growth and development of cellular elements was performed at 3, 7, 10, 14 days.

At the $7^{\text {th }}$ day of experiment (Figure 5) in all experimental samples three areas of growth were formed: compact, consisting of spindle cells and polygonal shapes, network like one, consisting of bundles and strands of cells, and area of migrating isolated cells.

In vials of PU samples containing folate-lactose wider areas of growth compared with the control were observed, there was tissue like growth.

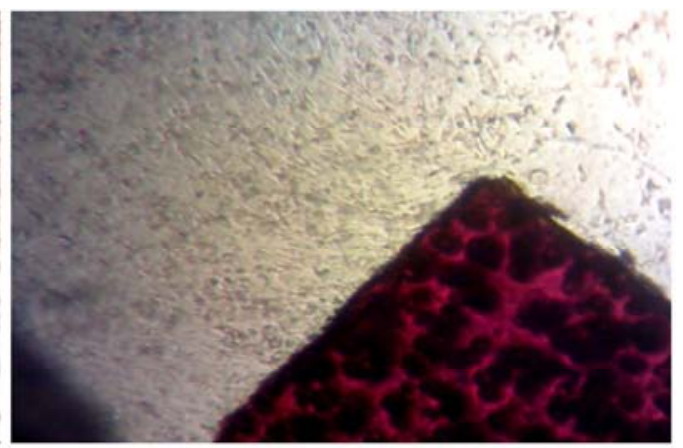

b

Figure 5. 7th day of experiment: the growth of fibroblasts in culture with control samples (a); tissue like growth in culture with samples of polyurethane containing folate-lactose (b).

Degeneration changes in control samples started at the $10^{\text {th }}$ day of experiment in compact area of the growth.

After 14 days (Figure 6) of cultivation the growth zone of cellular elements in the experimental vials differed from control cultures. In the control cell population was entering a phase of severe degeneration, manifested in large cytoplasm

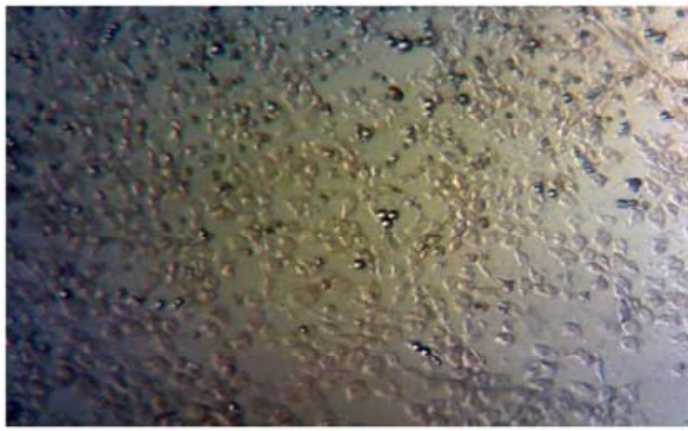

a vacuolization and granular degeneration of cells. In the experimental samples degenerative changes were found only in a compact area of the growth. Complete degeneration of all growth zones in experimental samples was observed at the $21^{\text {th }}$ day of experiment.

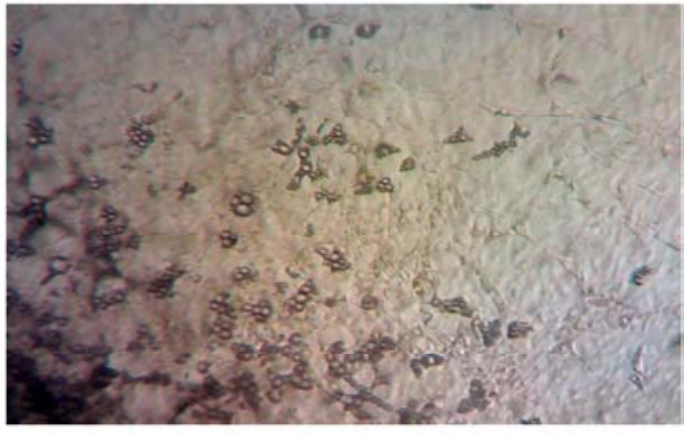

b

Figure 6. 14th day of experiment: degenerative changes in culture with control samples (a); continued growth of fibroblasts in culture with samples of PU with folate-lactose (b).

Our studies indicate that the culture of fibroblasts in control and in experimental samples are in the stage of stable growth. For samples of PU containing folate-lactose more active growth of fibroblastic elements than in control samples for 7-10 days was observed. Presence of folate-lactose slowed down the process of degeneration fibroblastic elements compared with the control samples indicating that investigated samples are biologically active and biocompatible.

\subsection{Histological Tests}

At $7^{\text {th }}$ day after implantation around polymeric samples DPP-lactose, the difference between the implanted material and the surrounding tissues is observed due to the formation of a wide leukocyte shaft. Among the white blood cells, neutrophils that actively absorbed the products of destroyed tissues prevailed. Other types of leukocytes - lymphocytes and monocytes were present in small amounts. A 
characteristic feature of the historical picture at this term of incubation was the presence of a large number of tissue macrophages - histiocytes, which were transformed from monocytes of blood and actively phagocyted neutrophilic detritus, clearing the zone of injury and preparing it for

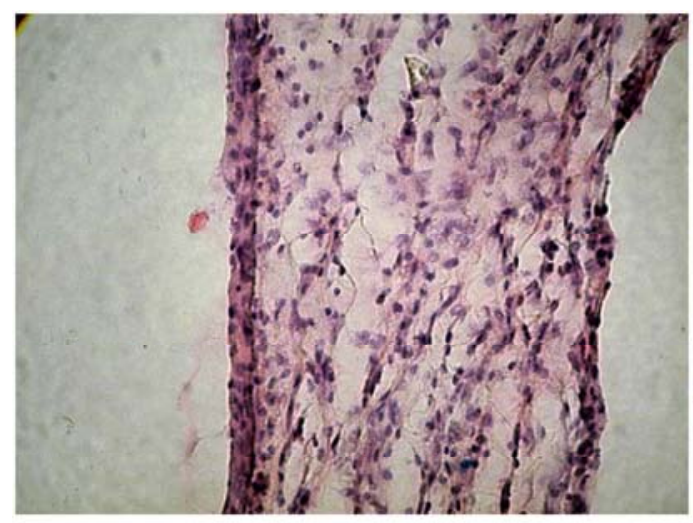

a proliferative processes (Figure 7, a). On separate areas, nonmatures fibroblastic elements were observed without clear signs of their maturity, as well as less differentiated cellular elements. Blood vessels were presented in a small number without disturbing the microcirculatory processes in them.

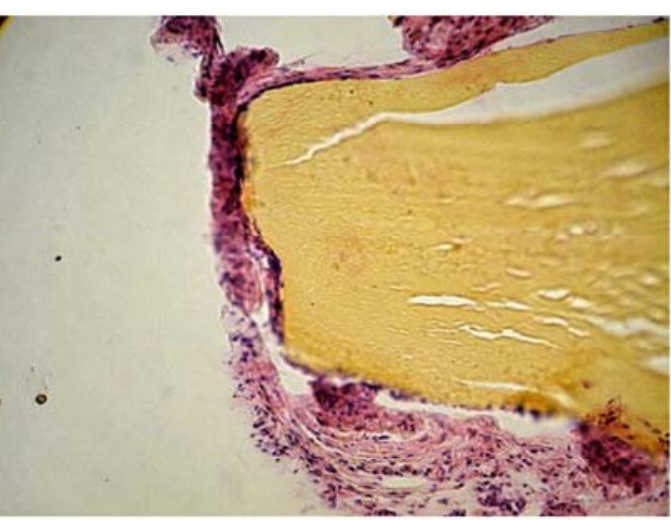

b

Figure 7. The expressed leukocyte and macrophage reaction around the implanted DPP-lactose sample at day 7 of the experiment (a). Coloring with hematoxylin and eosin. $\times 100$; Weak in intensity leukocyte and macrophage infiltration around the implanted sample of DPP-folate lactose on 7 th day of experiment (b). Coloring with hematoxylin and eosin. $\times 100$.

At $7^{\text {th }}$ day after implantation around the polymeric samples DPP-folate lactose there were initial processes of formation of connective tissue capsule, which was characterized by different degree of maturity. So, in some areas, there was a rather mature capsule, consisting of spindle-shaped fibroblasts that were between the rows of beams of collagen fibers. In other areas, there were mild-differentiated cell elements located on the border of implant-capsule. Throughout the length of the capsule there was a weak in intensity leukocyte and macrophage infiltration (Figure 7, b). Single lymphocytes were observed. Blood vessels were presented in a small number, without signs of disturbance of microcirculation in them.

At 14th day after surgery around the DPP-lactose polymer samples, decrease in the thickness and density of the leukocyte shaft compared to the previous study period was observed. Instead of that, the amount of cellular detritus increased, which led to increase in the intensity of the monocyte-macrophage reaction (Figure 8 , a), which was the evidence of active phagocytic processes. The connective tissue capsule was insufficiently mature at this time of study, although on some areas, young forms of fibroblast cells and single spindle-shaped fibroblasts were observed. The insignificant exudation of tissues surrounding the capsule was observed, as well as their lymphocytic infiltration. The number of blood vessels increased significantly, their lumen increased, some of them were characterized by the presence of marginal standing of red blood cells, but without elements of stasis and thrombosis.

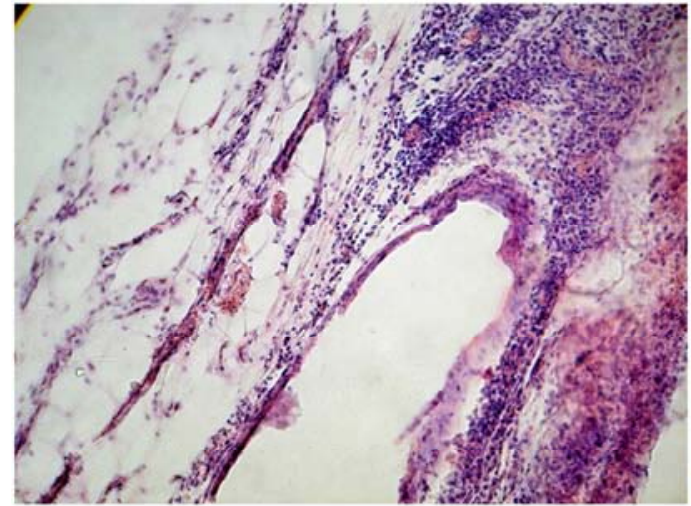

a

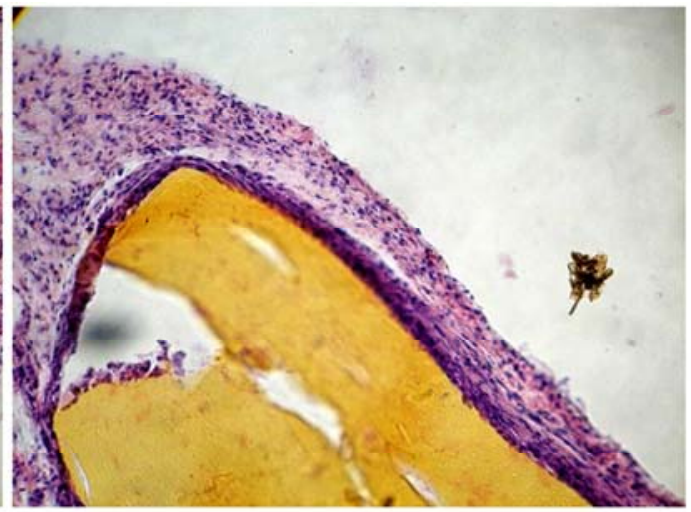

b

Figure 8. Mature connective tissue capsule with a large amount of blood vessels around the implanted DPP-lactose sample at day 14 of the experiment (a). Coloring with hematoxylin and eosin. $\times 100$; Connective tissue capsule around the implanted DPP-folate lactose sample at day 14 of the experiment (b). Coloring with hematoxylin and eosin. $\times 100$.

At $14^{\text {th }}$ day after the operation around the polymer specimens DPP-folate lactose, less marked cellular reactions were observed than around the DPP-lactose sample. A mature and thin connective tissue capsule, consisting of spindleshaped fibroblasts, located between rows of beams of collagen fibers, was observed. In separate areas there was an 
insufficiently mature capsule consisting of young forms of fibroblast cells, leukocyte infiltration, as well as a large number of monocytic-macrophage cells with phagocytic activity (Figure $8, \mathrm{~b}$ ). Blood vessels were presented in a small number without violations of microcirculatory processes in them.

30 days after the operation around the DPP-lactose sample, a thick, mature connective tissue capsule was observed. The inner layer of the capsule was represented by a large number of monocyte-macrophage elements, with insignificant leukocyte and lymphocytic infiltration. Bundles of collagen fibers were located around and spindle-shaped fibroblasts between them (Figure 9, a), which actively synthesized collagen. At this period of the study there was a normalization of microcirculatory processes in the blood vessels, compared with the previous term of the study.

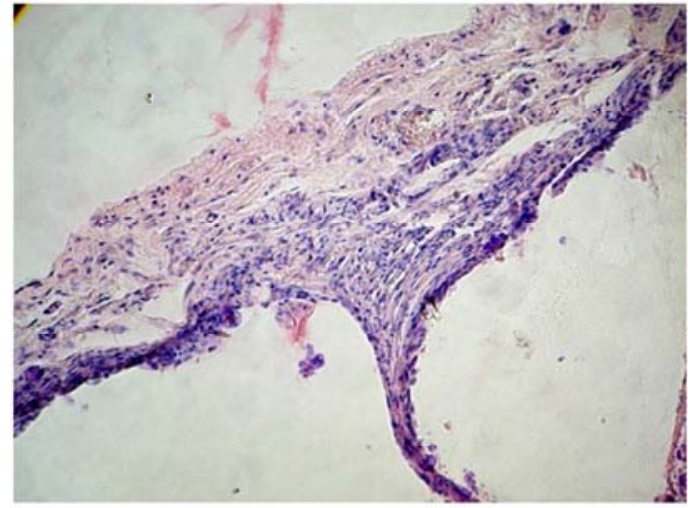

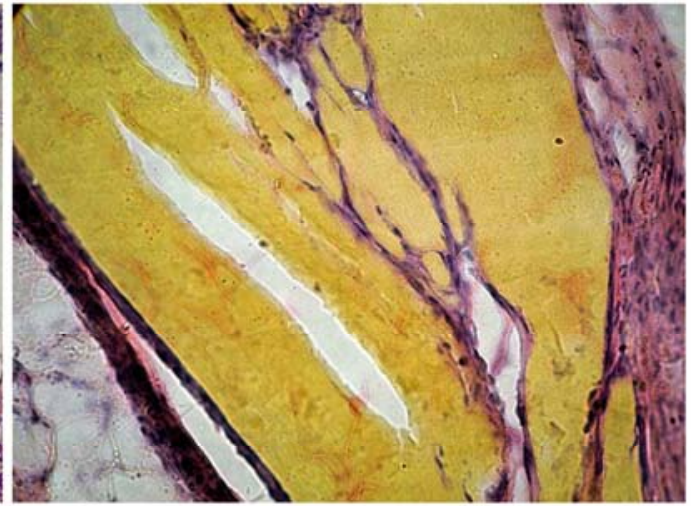

b

Figure 9. A thick connective tissue capsule around the implanted DPP-lactose sample at 30 days of the experiment (a). Coloring with hematoxylin and eosin. $\times$ 100; Mature connective tissue capsule and insignificant macrophage infiltration around the implanted DPP-folate lactose sample at 30 days of the experiment (b). Coloring with hematoxylin and eosin. $\times 100$.

30 days after surgery around the DPP-folate lactose sample, a mature and fairly thin connective tissue capsule, consisting of bundles of mature collagen fibers and spindleshaped fibroblasts between them, oriented along implanted materials, was observed (Figure 9, b). At this time of study, the intensity of the monocyte-macrophage reaction slightly decreased, leukocyte and lymphocytic infiltration were insignificant. Blood vessels were presented in a small amount, without violations of microcirculatory processes in them.

90 days after the surgery around the DPP-lactose sample,

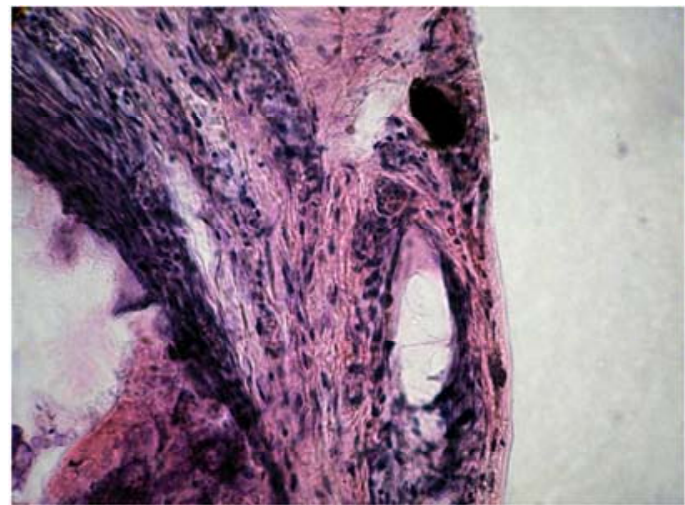

the connective tissue capsule had a high degree of maturity throughout its length (Figure 10, a). Spindle-like fibroblasts located between the beams of wavy collagen fibers, oriented along the implanted sample, were the main cellular elements of the capsule. The monocytic-macrophage reaction remained quite pronounced, but decreased, compared with the previous study term. Leukocyte and lymphocytic infiltration were insignificant. The number of blood vessels decreased, compared with the previous terms of study, due to their reduction. Single vessels were characterized by normal microcirculatory processes.

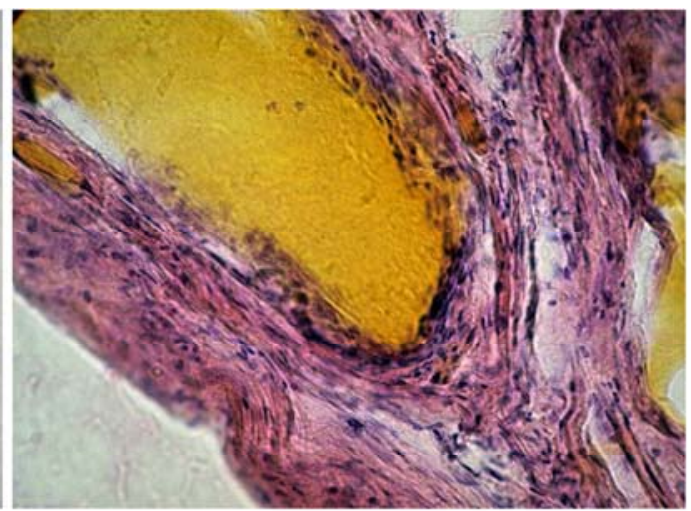

b

Figure 10. Connective tissue capsule around the implanted DPP-lactose sample for 90 days of the experiment (a). Coloring with hematoxylin and eosin. $\times$ 100; Connective tissue capsule with intense macrophage reaction around the implanted DPP-folate lactose sample at 90 days of the experiment (b). Coloring with hematoxylin and eosin. $\times 100$.

90 days after the surgery around the DPP-folate lactose sample, there was a mature and thin connective tissue capsule, the main cellular elements of which were spindle- like fibroblasts lying between the beams of wavy collagen fibers. At the same time, increase in the intensity of the monocyte-macrophage reaction was observed (Figure 10, b), 
which is possibly due to the processes of biodegradation of the polymer sample and the active participation of macrophages in the phagocytosis of the polymer fragments. The number of blood vessels was insignificant, some of them had an enlarged lumen. Violations of microcirculatory processes in the blood vessels was not recorded.

Thus, conducted histological studies have shown that there already was separation of implanted polymer samples from surrounding tissues in the early stages of the study. The cellular reactions observed around the implanted polymer samples of DPP-lactose and DPP-folate lactose were typical for aseptic inflammation and did not lead to the development of acute and chronic inflammatory processes in the organism of experimental animals.

\section{Conclusions}

Biologically active PU based on diisocyanate prepolymer and folate-lactose with improved performance has been synthesized. A number of physical-mechanical, thermophysical and medical-biological researches of the prepeared polymeric materials have been carried out. The value of the strength at break and elongation of polymer material samples with folate-lactose are higher by $83 \%$ and $22 \%$ than the corresponding physical and mechanical properties of the polymer material with D-lactose. According to the obtained results, the characteristic viscosity and water absorption of polyurethane containing folate-lactose are higher by $44 \%$ and $34 \%$ for the parameters of polyurethane containing D-lactose.

It has been established that prepeared PU are singlephase. The glass transition temperature is $247 \mathrm{~K}$ for PU with FL and $245 \mathrm{~K}$ for PU with D-lactose. At the same time, folate-lactose introduction into the polymer matrix causes decreasion of the heat jump value $(\Delta \mathrm{Cp})$ to $0,1551 \mathrm{~J} / \mathrm{g}\left({ }^{\circ} \mathrm{C}\right)$ for PU with FL sample and $0,2911 \mathrm{~J} / \mathrm{g}\left({ }^{\circ} \mathrm{C}\right)$ for PU with Dlactose sample.

Biodegradation of synthesized materials was investigated. According to research results, polyurethane, modified by folate-lactose, retains sufficient performance for use within 6 months in conditions close to the human body.

It was shown, that samples of a polymer with folatelactose exhibit biological activity by slowing the degradation of fibroblasts under tissue culture conditions by method of tissues culture examinations.

Histologic studies have given an opportunity to study the effect of polymer compositions with lactose and folate lactose on tissues, which surrounding implants. Thus, the introduction of folate lactose into the structure of the polymeric implant led to a more rapid maturation of the connective tissue capsule, less circular cell infiltration in the capsule was observed at all stages of the study, indicating the stimulation of the regeneration processes under the influence of folate lactose in the polymer matrix. Thus, the biological activity of the implants with folate lactose was confirmed by tissue culture and histological studies.

\section{References}

[1] N. A. Galatenko, R. A. Rozhnova, O. S. Andryushina Pat. 55891 UA, C 08G 71/00; C 07D 475/00; A 61L 31/00. "Diamine-containing polyurethaneureas with folic acid as polymer film biologically active materials medical purpose", № u201008173; appl. 30.06.10; publ. 27.12.10.

[2] J. A. Braatz, C. L. Kehr. Pat. 4886866 USA, C 08G 18/10. "Contact lenses based on biocompatible polyurethane and polyurea-urethane hydrated polymers", No. 312.331; appl. 16.02.1989; publ. 12.12.1989.

[3] Xiangyu Liu, Yuqing Niu, Kevin C. Chen, Shiguo Chen. "Rapid hemostatic and mild polyurethane-urea foam wound dressing for promoting wound healing", Materials Science and Engineering: C, 2017, Vol. 71, pp. 289-297.

[4] Gubskij Yu. I. Biological Chemistry: Book / Gubskij Yu. I.. Kiyv - Ternopil: Ukrmedkniga, - 2000. - 508 p.

[5] A. K. T. Thulasidasan, A. P. Retnakumari, M. Shankar, V. Vijayakurup, S. Anwar, S. Thankachan, K. S. Pillai, J. J. Pillai, C. D. Nandan, V. V. Alex, T. J. Chirayil, S. Sundaram, G. S. V. Kumar, R. J. Anto (2017). Folic acid conjugation improves the bioavailability and chemosensitizing efficacy of curcuminencapsulated PLGA-PEG nanoparticles towards paclitaxel chemotherapy. Oncotarget 8 (64), 107374-107389.

[6] Z. He, J. Huang, Y. Xu, X. Zhang, Y. Teng, C. Huang, Y. Wu, X. Zhang, H. Zhang, W. Sun (2015). Co-delivery of cisplatin and paclitaxel by folic acid conjugated amphiphilic PEGPLGA copolymer nanoparticles for the treatment of non-small lung cancer. Oncotarget 6 (39), 42150-42168.

[7] Min-Hua Chen, Chung-King Hsu, Feng-Huei Lin, L. Stobinski, J. Peszke (2006). Folic acid immobilized ferromagnetic DP-bioglass to target tumor cell for cancer hyperthermia treatment. Advances in Science and Technology 53, 50-57.

[8] J. Zhang, S. Rana, R. S. Srivastava, R. D. Misra. On the chemical synthesis and drug delivery response of folate receptor-activeted, polyethylene glycol-functionalized magnetite nanoparties (2008). Acta Biomaterialia 4, 40-48.

[9] K. Butzbach, M. Konhäuser, M. Fach, D. N. Bamberger, B. Breitenbach, B. Epe, P. R. Wich. Receptor-mediated Uptake of Folic Acid-functionalized Dextran Nanoparticles for Applications in Photodynamic Therapy. Polymers 2019, 11 (5), 896.

[10] Qing Liu, Wenjing Guo, Mei Yang, Kejie Wang, Weijun Liu, Fanhong Wu. Release. Behavior of Folic Acid Grafted Hollow Hydroxyapatite as Drug Carrier. Advances in Polymer Technology, Volume 2019, Article ID 9562437, 9 pages.

[11] O. S. Andrushina, R. A. Roznova, N. A. Galatenko, T. O. Kiselova. The Synthesis of New Polyurethane Ureas with Folic Acid (2010). Polymer Journal (18181724) 32, 84-88.

[12] N. Galatenko, R. Rozhnova, L. Kulyk, D. Kulesh. The evaluation of biocompatibility and biological activity of composite materials with folate-derivative of ferrocene for medidine (2016). Chemistry, physics and technology of surface 7 (3), 344-353.

[13] L. V. Kulyk, I. I. Gladyr, R. A. Rozhnova, N. A. Galatenko. The New biological active polyurethane with folate-lactose in own structure: synthesis and properties (2015). Ukrainian Chemical Journal 81 (8), 117-121. 
[14] Siggia S. and Hanna J. G.: Quantitative Organic Analysis via Functional Groups. Moscow: Khimiya, 1983. [in Russian].

[15] Bellamy, L. The Infra-red Spectra of Complex Molecules. Moscow: Izdatelstvo inostrannoj literatury, 1957 [in Russian].
[16] Heinz Becker, Werner Berger, Günter Domschke Bekker G. Organicum: Practical Handbook of Organic Chemistry. Moscow: Myr, Vol. 1, 1979 [in Russian]. 